\title{
Les enjeux politiques et de gestion des ressources humaines de l'évaluation des professeurs en France
}

\author{
Hervé Kéradec \\ Inspecteur d'Académie \\ Membre du CNIRÉ ${ }^{1}$
}

Résumé : L'évaluation des professeurs dans le système éducatif français est souvent critiquée, néanmoins les pratiques d'évaluation ont peu changé depuis 1972. Cet article interroge la dimension politique et la dimension de gestion des ressources humaines de cette évaluation. Il montre que l'évaluation des professeurs est d'abord une question politique, qu'au-delà des quelques travaux de recherche, des divers rapports et des circulaires officielles, les choix actuels résultent d'un compromis entre les forces syndicales et ministérielles en présence. Plusieurs pistes sont proposées pour rendre l'inspection plus efficace, mettre en place un vrai développement professionnel des professeurs et de l'encadrement, valoriser les meilleurs professeurs et accompagner les enseignants en difficulté; sachant que seule une volonté politique forte permettra la mise en œuvre effective de tels changements.

Mots clés : évaluation des professeurs, inspection, développement professionnel, formation, gestion des ressources humaines, politiques d'éducation, système éducatif français.

\footnotetext{
${ }^{1}$ Conseil National de l'Innovation pour la Réussite Éducative. Ce conseil, créé en 2013, est placé auprès de la ministre de l'éducation nationale, de l'enseignement supérieur et de la recherche.
} 


\section{Introduction}

L'évaluation des professeurs est une question vive, qui a suscité d'importants mouvements de protestation en France en 2012, alors qu'elle faillit être très bouleversée, mais avant que l'alternance politique conduise à laisser les choses en l'état. Cette recherche interroge donc ce paradoxe apparent d'une critique régulière des modalités d'évaluation des professeurs - de la visite de l'inspecteur, du rapport, des notes, de l'évaluation par le chef d'établissement - et de la très grande stabilité institutionnelle des procédures en place.

Une inspection collective des établissements et des disciplines ne pourrait-elle pas remplacer l'inspection individuelle? Comment faire de l'inspection individuelle un vrai facteur de changement des pratiques? Comment valoriser les bons professeurs et accompagner les enseignants en difficulté ? Pourra-t-on finalement modifier l'évaluation des professeurs en France? Nous examinerons ces questions en prenant appui sur un corpus de recherches finalement peu nombreuses au regard de l'importance de la question. Il ne semble pas que les sciences de l'éducation se passionnent pour cette question, en revanche le ministère de l'Éducation nationale revient régulièrement à cette problématique, en demandant des rapports (HCÉÉ ${ }^{2}, 2003$; Pochard, 2008; IGEN ${ }^{3}$, 2013) et en produisant régulièrement des textes officiels $(2005,2009,2015)$ sur les missions des inspecteurs. Nous prendrons aussi appui sur notre propre démarche de praticien réflexif, qui depuis treize années, s'efforce de cerner une pratique d'une grande complexité, mais déterminante pour l'amélioration de la qualité de l'enseignement. Nous analyserons d'abord les enjeux politiques de l'évaluation des professeurs avant d'en sérier les enjeux de gestion des ressources humaines (GRH), et de tracer des pistes de renouvellement des pratiques françaises en la matière.

\section{Les enjeux politiques de l'évaluation des professeurs}

L'inspection des professeurs est au cœur de leur évaluation. C'est loin d'être une question mineure car elle détermine très largement le rythme de déroulement de leur carrière. Curieusement elle donne lieu à peu de publications scientifiques, comme nous l'avons constaté lors d'une recherche bibliographique conduite avec l'aide du centre de documentation ${ }^{4}$ de l'Ecole supérieure de l'éducation nationale de l'enseignement supérieur et de la recherche (ESENESR) une seule thèse (Albanel, 2007) et quelques articles sont disponible sur l'inspection individuelle des professeurs. Les chercheurs interrogés à ce sujet soulignent que les corps d'inspections ne faciliteraient pas les enquêtes sur cette question et les inspecteurs, pour leur part, affirment qu'ils ne sont jamais sollicités par les chercheurs... En revanche le ministère redéfinit régulièrement ce qu'il attend de l'évaluation des professeurs, par touches successives, en amendant le texte précédent et en mettant l'accent sur

\footnotetext{
${ }^{2}$ Haut Comité pour l'Évaluation de l'École.

${ }^{3}$ Inspection générale de l'Éducation Nationale.

${ }^{4}$ Nous remercions en particulier madame Nicole Siebert-Taabni, responsable du centre de ressources de l'Ecole Supérieure de l'Éducation Nationale de l'Enseignement Supérieur et de la Recherche (ESENESR), pour son aide dans cette recherche des sources scientifiques.
} 
tel ou tel point. Selon la doctrine du ministre en exercice on constate certaines évolutions que nous étudierons en analysant les trois textes les plus récents - 2005, 2009, 2015- dont la juxtaposition est très éclairante sur l'évolution des missions d'évaluation que l'institution assigne aux inspecteurs.

\subsection{Note de 2005 : inspection individuelle et collective}

Le texte de $2005{ }^{5}$ pose avec beaucoup de clarté la place de l'inspection individuelle des professeurs et en définit précisément les contours : «La mission d'évaluation des inspecteurs qui constitue le cœur de leur métier concerne d'abord l'acte d'inspection individuelle des enseignants et autres personnels placés sous leur responsabilité. Cette inspection est importante dans ses dimensions de contrôle et de conseil et pour la prise en compte de la compétence et de l'engagement de l'enseignant aux différentes étapes de sa carrière et dans le respect des initiatives pédagogiques appropriées. L'inspection apprécie les activités et compétences des personnels dans leur domaine propre, le respect des programmes et leur efficacité dans les apprentissages. Elle prend également en compte leur implication dans le fonctionnement général de l'école, de l'établissement et de l'institution. L'inspection individuelle comprend un entretien. Le résultat se matérialise par un rapport écrit, base d'une note.» Ce texte décrit avec précision le travail d'évaluation à conduire. Notons que le passage consacré à l'évaluation des professeurs arrive en premier, avant les autres missions d'animation et d'impulsion, de formation et d'expertise. Ce texte de Paul Desneuf, directeur de l'encadrement de l'époque, prévoit d'autres formes d'inspection, plus collectives : « $A u$ delà de l'acte d'inspection individuelle, les inspecteurs doivent, sous l'impulsion du recteur, procéder l'évaluation des enseignements et des établissements. Inspections croisées ou globales permettent ainsi d'avoir une connaissance approfondie de l'état des disciplines et activités de l'établissement ou école, du respect des programmes, de l'application et de l'adéquation des réformes et de proposer les interventions et aménagements nécessaires. » On voit poindre une autre type d'évaluation des établissements et des disciplines qui sera pratiquée de manière inégale suivant les académies, car elle s'avérera très gourmande en temps et d'une mise en œuvre complexe.

\subsection{Circulaire de 2009 : maitrise disciplinaire et acquis des élèves}

La circulaire de $2009^{6}$, signée Roger Chudeau, directeur de l'encadrement de l'époque, structure la mission des inspecteurs autour du pilotage, du management, et du conseil. La langue ce cette circulaire se veut résolument moderne et managériale, le terme de management, généralement peu apprécié des professeurs et des syndicats y fait même son entrée. «La compétence et la légitimité des corps d'inspection sont fondées sur la maîtrise de leur discipline ou de leur spécialité respective. Les inspecteurs procèdent à l'évaluation des

\footnotetext{
${ }^{5}$ Note de service $n^{\circ} 2005-089$ du 17-6-2005, publiée au Bulletin officiel de l'éducation nationale, $n^{\circ} 25$ du 30 juin 2005.

${ }^{6}$ Circulaire n ${ }^{\circ}$ 2009-064 du 19-5-2009, Ministère de l'Education Nationale, publiée au Bulletin officiel de l'éducation nationale $\mathrm{n}^{\circ} 22 \mathrm{du} 28$ mai 2009.
} 
enseignements, des écoles et établissements ou à celle de champs disciplinaires ou éducatifs : L'inspection individuelle des enseignants dans leur classe est essentielle pour vérifier la qualité de l'enseignement dispensé. Elle l'est aussi pour assurer le pilotage de l'action éducative dans l'académie. Elle permet une observation fine et une connaissance approfondie de l'état des disciplines et des activités de l'école ou de l'établissement. Elle vérifie le respect des programmes, l'application des réformes et mesure l'efficacité de l'enseignement dispensé en fonction des résultats et des acquis des élèves. » La dimension disciplinaire est nettement rappelée et posée comme source de légitimité de la mission de l'inspecteur. Le second point saillant de ce texte est la notion d'acquis des élèves qui sont clairement posés comme l'objectif de l'enseignement, en lien avec le respect des programmes. L'inspection individuelle est rappelée comme pratique essentielle de l'évaluation des professeurs, mais complétée dans une dimension de pilotage : «Toutefois, l'évaluation d'équipes disciplinaires ou pédagogiques, l'évaluation de niveaux ou de cycles, l'évaluation systémique d'unités éducatives, sont des formes d'interventions qui viennent désormais placer l'inspection individuelle dans une perspective de véritable pilotage pédagogique. ${ }^{7}{ }^{\prime}$

Ce texte, très coloré d'approche systémique, introduit la notion de pilotage pédagogique sans en donner un sens précis. Le mot pilotage, dans le lexique de la théorie des systèmes organisationnels est donné aux décisions qui ne sont ni de régulation, ni de planification, c'est le niveau intermédiaire du management, la planification relevant du ministère et la régulation exprimant l'action des professeurs et de l'encadrement au quotidien ${ }^{8}$. Le texte précise alors : «Ces formes d'interventions sont d'ailleurs aisément combinées avec des inspections individuelles. Elles peuvent aussi revêtir la forme d'audits interdisciplinaires, où l'inspection est un des éléments d'une évaluation plus globale. Ce type d'intervention est déclenché par l'autorité académique et a vocation à être systématisé. ${ }^{9}$ "Il s'agit bien de l'affichage d'une volonté politique de développer d'autres formes d'évaluation plus collectives en faveur d'un management moderne articulant évaluation individuelle et évaluation collective. Dans la pratiques ces évaluations seront assez rares et cette nouvelle forme de management ne sera plus vraiment portée après le départ du directeur de l'encadrement (en poste du 26 septembre 2008-13 octobre 2010) en et la suppression de cette direction au profit de la seule direction des ressources humaines.

Dans la partie titrée «management» de cette circulaire, on revient sur l'inspection individuelle: « L'inspecteur conçoit ses interventions directes auprès du personnel enseignant comme un acte de gestion de la ressource humaine et éducative de l'académie. L'avis de l'inspecteur est sollicité par l'autorité académique dans tous les grands actes de gestion des personnels : titularisation, évaluation, avancement, promotion et affectation. ${ }^{10}{ }$ La logique en place est stipulée comme une démarche de gestion de ressources humaines, la GRH, terme du lexique managérial, auquel on a ajouté éducative pour ne pas trop froisser les susceptibilités syndicales.

\footnotetext{
${ }^{7}$ Ibidem.

${ }^{8}$ On pourra lire sur ce point J-L. Le Moigne (1974), Les systèmes de décision dans les organisations, Paris, PUF.

${ }^{9}$ Circulaire ${ }^{\circ}$ 2009-064 du 19-5-2009 Ministère de l'Education Nationale, publiée au Bulletin officiel de

l'éducation nationale ${ }^{\circ} 22$ du 28 mai 2009.

${ }^{10}$ Ibidem.
} 


\subsection{Circulaire de 2015 : accompagnement et pilotage pédagogique}

La circulaire de décembre $2015^{11}$, s'inscrit dans le contexte tendu de la réforme du collège devant être mise en place en septembre 2016. Ce texte rappelle surtout aux inspecteurs leur rôle dans la mise en place des réformes et attend d'eux une implication sans faille dans cette mission. Il se veut en rupture par rapport au texte de 2009 et parle de «reprécisions » du travail des inspecteurs : "L'accompagnement individuel et collectif d'une part, le pilotage pédagogique d'autre part, constituent désormais le cour de la mission des personnels d'inspection. Dès lors, leur rôle et leur place en académie doivent être reprécisés. ${ }^{12}{ }^{2} \mathrm{Le}$ terme inspection disparait, ainsi que le terme d'évaluation, la mission étant principalement centrée sur l'accompagnement et le pilotage pédagogique qui sont précisés plus avant. Finalement l'inspection individuelle y occupe une place très restreinte, le texte précise :

«Dans le cadre de leur mission d'inspection, les inspecteurs s'assurent de la qualité de l'enseignement dispensé au regard des apprentissages et acquis des élèves. L'inspection individuelle, si elle répond au besoin de gestion de la carrière des personnels, a pour objectif principal l'accompagnement et la formation des personnels enseignants, d'éducation et d'orientation. $^{13}{ }^{~}$ Dans le prolongement de la circulaire précédente, l'accent porte sur les acquis des élèves, il n'est fait aucune mention des compétences disciplinaires des inspecteurs, mais on peut en inférer que ces compétences sont implicites pour aider les élèves à construire leurs acquis. L'évaluation semble de faible importance dans ce texte, alors que dans la pratique l'inspection individuelle conduit toujours à donner une note qui déterminera le rythme d'avancement dans la carrière, ainsi que la production d'un rapport qui sera déterminant pour la suite de la carrière du professeur. La phrase «si elle répond au besoin de gestion de la carrière des personnel» signifie clairement que l'inspection individuelle n'est plus posée comme la mission centrale des inspecteurs. Deux points sont mis en avant: l'accompagnement des professeurs et leur formation. La pratique d'accompagnement gagnerait d'ailleurs à être précisée. Beaucoup d'autres missions sont confiées aux inspecteurs par cette circulaire : « formation initiale et continue, gestion des ressources humaines, contribution à la définition de la stratégie académique, impulsions des réformes et animation des équipes, évaluations des enseignements et des unités d'enseignement, conseils aux recteurs, missions nationales et en lien avec les collectivités territoriales. ${ }^{14} \gg$. Par la suite l'évaluation apparaît à nouveau dans le texte non pas forme d'évaluation individuelle mais sous forme d'évaluation collective des établissements, et de suivi des procédures de contractualisation entre les établissements et l'académie.

Pour comprendre l'esprit de ce texte il faut rappeler un élément de contexte très éclairant : la réforme du collège. La ministre, dans le cadre de la loi de rénovation de l'école a décidé d'introduire de nouveaux enseignements, les enseignements pratiques interdisciplinaires (EPI), de développer l'accompagnement personnalisé et d'opérer différents changement dans

\footnotetext{
${ }^{11}$ Circulaire du ministère de l'éducation nationale de l'enseignement supérieur et de la recherche $n^{\circ} 2015-207$ du 11-12-2015, publiée au bulletin officiel de l'éducation nationale de l'enseignement supérieur et de la recherche $n^{\circ} 47$ du 17 décembre 2015, cette circulaire abroge et remplace la circulaire $n^{\circ} 2009-064$ du 19 mai 2009.

12 Ibidem.

${ }^{13}$ Ibidem.

${ }^{14}$ Ibidem.
} 
l'enseignement des langues anciennes et des langues étrangères. En décembre 2015, les choses se précisent est les formations des professeurs sont annoncées pour la rentrée de janvier 2016, la mobilisation syndicale contre la réforme s'exprime assez fortement depuis la rentrée de septembre 2015. Dans ce contexte, la circulaire de décembre, gomme de plus en plus les aspects disciplinaires de la mission des inspecteurs pour la centrer sur l'accompagnement et le pilotage pédagogique, dont il est attendu qu'ils facilitent la mise en place de cette réforme.

\subsection{Synthèse sur une décennie de doctrines d'usage des corps d'inspection (2005-2015)}

L'examen de ces trois textes est riche d'enseignements sur la manière dont le ministère français en charge de l'éducation conçoit l'évaluation des professeurs. Il nous faut aussi souligner que nous n'avons pas repris, dans cet article, le texte d'un décret du 7 mai 2012, pris par le ministère Chatel, au lendemain de l'élection du président de la République le 6 mai 2012, qui marquait une alternance politique et conduisait automatiquement au départ de ce ministre. Ce texte prévoyait de confier l'évaluation des professeurs aux chefs d'établissement dont les fonctions managériales étaient fortement renforcées. Ce décret a été immédiatement abrogé par Vincent Peillon nouveau ministre de l'Éducation nationale pour revenir au précédent décret ${ }^{15}$. Trois tendances majeures se dégagent de ces trois textes: le développement du collectif, le déclin du disciplinaire, le primat du politique.

Le texte de 2005 insiste sur les inspections d'établissement et les inspections croisées, l'inspection doit aussi évaluer la dynamique de travail au sein de l'établissement, et l'on veut s'éloigner d'une conception trop personnelle de la compétence enseignante, donner plus d'importance au projet d'établissement et inscrire l'action du professeur dans une dynamique collective. Cette tendance se trouve confortée dans les textes de 2009 et 2015.

Le déclin du disciplinaire est surtout affiché dans la circulaire de 2015, en lien avec la mise en place des enseignements pratiques interdisciplinaires (EPI). La compétence des inspecteurs est d'abord posée comme didactique et pédagogique sans que mention soit faite de la compétence disciplinaire - scientifique, littéraire ou artistique. La faible place accordée au disciplinaire exprime la volonté de développer le travail interdisciplinaire et la participation de l'enseignant au projet d'établissement lui-même inscrit dans le projet académique.

Le texte de 2015 exprime une politique volontariste ; la mission des inspecteurs est de servir de relai à la «mise en ouvre effective » de la loi de refondation de l'école, à « (...) expliciter le sens des reformes, contribuer à leur application au plus près des personnels (...)». Cette circulaire a le mérite d'exprimer clairement les priorités de la rentrée 2016 et atteste de la grande cohérence entre les attendus de la réforme du collège et les missions assignées au corps d'inspection pour sa mise en œuvre.

\footnotetext{
${ }^{15}$ On pourra lire sur ce sujet : Kéradec H. (2013) Herméneutique d'un décret mort-né, In Actes du colloque “Les question vives en éducation et formation : regards croisés France-Canada ". Nantes : 5-7 juin 2013 (actes en ligne : http://www.cren.univ-nantes.fr/)
} 
Ces trois textes fondamentaux révèlent les enjeux de l'évaluation des professeurs du point de vue de sa direction politique et administrative, il importe aussi de s'interroger sur les l'impact en termes de GRH de l'évaluation des professeurs.

\section{Les enjeux de gestion des ressources humaines}

Par-delà les choix politiques et d'orientation du système éducatif qui s'expriment dans la parole ministérielle, l'évaluation des professeurs est un acte majeur de gestion des ressources humaines, que nous étudierons suivant trois modalités : recruter, promouvoir, former. Nous n'aborderons pas la modalité rémunérer, comme cela se fait généralement en GRH, car celleci est totalement dépendante, dans l'administration française, du recrutement dans un corps et de la promotion en son sein.

\subsection{Recruter}

Le recrutement par concours est le seul qui permet de rejoindre un corps d'enseignement de l'Etat français et de devenir fonctionnaire titulaire. Les autres enseignants sont vacataires et contractuels, ils sont recrutés directement par les inspecteurs et ont un statut précaire. Nous ne développerons pas longuement la question des concours qui pourrait faire l'objet d'un travail de recherche spécifique. Les professeurs qui présentent un concours passent des épreuves d'admissibilité et d'admission, dont les formes sont multiples suivant les concours et ont beaucoup évoluées au fil des années, mais dont les fondements démocratiques républicains restent conformes aux principes des structures bureaucratiques au sens de Max Weber ${ }^{16}$. Ces concours de recrutement des professeurs agrégés et certifiés respectent les principes de rationalité, d'égalité d'accès et de classement qui fondent le recrutement de tout fonctionnaire de l'Etat. Après leur admission les professeurs du premier et du second degré intègrent une Ecole supérieure du professorat et de l'éducation (ESPÉ) et sont généralement intégrés dans le corps correspondant à leur concours, à la suite d'une première inspection dite de titularisation. Trois compétences particulières doivent être évaluées dans les concours de recrutement: disciplinaire, didactique et pédagogique. Historiquement la compétence disciplinaire est prégnante dans les concours, en particulier le concours de l'agrégation. L'aptitude à travailler avec des collègues n'est pas évaluée, certaines épreuves évaluent l'interrogation pédagogique du professeur et la pratique à conceptualiser sa pratique pour les candidats aux concours internes. La compétence didactique, c'est à dire l'interrogation sur les savoirs eux même et les obstacles à dépasser, au sens bachelardien (Bachelard, 1938) est très peu évalué ${ }^{17}$. Le recrutement par concours est inscrit dans les fondements des structures bureaucratiques wébériennes, elle ne saurait être remise en cause, mais la définition des épreuves peut changer, les modifications apportées résultant des rapports de pouvoirs entre acteurs de l'institution (Crozier et Friedberg, 1977).

\footnotetext{
${ }^{16}$ Max Weber, 1921.

${ }^{17}$ On pourra consulter sur ce point H. Kéradec, 2012, sur l'articulation nécessaire entre la didactique et l'épistémologie.
} 


\subsection{Promouvoir}

Depuis $1972^{18}$, la promotion des professeurs du second degré dépend d'un double évaluation, par un inspecteur, Inspecteur d'académie, Inspecteur pédagogique régional (IA-IPR) et par le chef d'établissement. Dans le premier degré les professeurs des écoles font l'objet d'une évaluation unique par l'Inspecteur de l'Éducation Nationale (IEN).

\subsubsection{L'évaluation par l'inspecteur}

$\mathrm{Au}$ cours de leur carrière les professeurs sont inspectés individuellement tous les quatre ou cinq ans et à l'occasion des grands événements de leur carrière, nomination sur un poste profilé, accès à la hors classe, demande de changement de corps... Pratiquement l'inspecteur d'académie, ou l'inspecteur de l'éducation nationale pour le premier degré, se rend dans l'établissement, assiste à une séance de cours, s'entretient avec le professeur, puis rédige un rapport et pose une note (Albanel, 2007) ${ }^{19}$.

L'observation d'une séance de cours est annoncée, de trois jours à une semaine à l'avance. Au fond de la classe, parfois accompagné du chef d'établissement - en lycée et collège -, l'inspecteur observe la séance de cours, pour évaluer, les qualités disciplinaires, didactiques et pédagogiques du professeur. Il évalue aussi la qualité de la relation à la classe, prend connaissances des traces ce cours, de la progression, des contrôles effectués, des copies corrigées et de toutes les informations que le professeur souhaite porter à sa connaissance.

L'entretien qui suit cette observation porte sur la carrière du professeur, son investissement dans ses enseignements, sa maitrise scientifique, sa réflexion didactique et pédagogique, son implication dans la vie de l'établissement et des sections qui lui sont confiées, sa capacité à travailler avec ses collègues et sa hiérarchie. Cet entretien est l'occasion de faire un bilan de carrière et d'examiner les perspectives d'évolution de carrière, de changement de poste, de répondre à des demandes spécifiques de formation ou de nomination à des fonctions particulières, dans les jurys de concours ou d'examen par exemple. L'entretien porte aussi sur la séquence qui vient d'être observée, avec l'appui de la fiche pédagogique produite par le professeur. Cet entretien permet d'évaluer la prise de distance du professeur par rapport à ses pratiques, sa connaissance de son public, son interrogation sur les acquis des élèves dans la séance et plus généralement dans l'ensemble de ses enseignements. Le climat dans lequel l'entretien se déroule doit être celui d'un accompagnement à la fois bienveillant et exigeant.

Un rapport d'inspection est rédigé après chaque visite et est envoyé à l'intéressé qui en prend connaissance, le signe, et qu'il est en droit de contester ; ce qui, dans la pratique, est rare. Cette contestation peut donner lieu à une nouvelle visite de l'inspecteur ou à un recours contentieux. Les rapports d'inspection sont conservés dans le dossier administratif du professeur et servent pour apprécier toute demande de promotion, ou de changement de poste

\footnotetext{
${ }^{18} \mathrm{Cf}$. Décret $\mathrm{n}^{\circ} 72-580$ du 4 juillet 1972 relatif au statut particulier des professeurs agrégés, et Décret $n^{\circ} 72-581$ du 4 juillet 1972 relatif au statut particulier des professeurs certifiés.

${ }^{19}$ Sur la pratique d'inspection dans l'enseignement secondaire, le principal travail réalisé à ce jour est la thèse de Xavier Albanel, 2007, qui a fait l'objet d'une publication en 2009.
} 
au cours de la carrière. Le rapport d'inspection souligne la qualité du travail réalisé par le professeur et propose des voies de progrès, des points d'amélioration possible de la réflexion et de la pratique. Les rapports sont généralement rédigés dans une langue nuancée et les silences du texte ne sont pas exempts de signification.

Une note est posée à la suite de la visite d'inspection. Cette note est très encadrée, elle est sur 60 points et dépend de l'échelon du professeur. La note situe de professeur dans une catégorie qui permet un avancement d'échelon à l'ancienneté, au choix ou au grand choix. L'appartenance à l'une ou l'autre de ces catégories conduit à un avancement de carrière plus ou moins rapide.

La visite d'inspection est aussi l'occasion de repérer un professeur pour lui confier des missions académiques ou nationales. Il peut lui être proposé de devenir formateur, tuteur de professeurs stagiaires, membre des commissions nationales d'examens et de concours, membre de jury de validation des acquis de l'expérience, membre des directoires d'examens etc. Ces nominations constituent un facteur de motivation pour le professeur, elles le valorisent, lui permettent de «sortir de son établissement », de rencontrer d'autres collègues et de s'investir dans de nouvelles missions.

\subsubsection{L'imaginaire de l'inspection}

La visite d'inspection est souvent ressentie comme stressante par le professeur (Albanel, 2009), et l'on peut observer que ce stress ne diminue pas avec l'âge. En France, la classe est un espace clos ou le professeur et les élèves forment une communauté singulière. Les seuls à pouvoir y pénétrer sont les inspecteurs, parfois accompagnés du chef d'établissement, et le stagiaire, quand le professeur est tuteur d'un professeur stagiaire. Il n'est pas de tradition que les professeurs puissent assister aux cours les uns des autres, même au début de leur carrière, comme cela est parfois souhaité par les formateurs des Espé. L'inspection est souvent vécue comme un moment infantilisant (Mosconi et Heideger, 2003). Cette situation peut rappeler au professeur quand il était écolier, et que le maître l'envoyait au tableau pour faire un exercice ou apporter un élément de correction, mêlant satisfaction narcissique et crainte d'échouer. La visite d'inspection est très souvent surinvestie affectivement, réactivant des schémas parent/enfant, au sens de l'analyse transactionnelle (Berne, 1958) qui ne facilite pas un travail objectif qui demanderait l'instauration d'une relation adulte/adulte. L'inspecteur est souvent perçu comme une figure d'autorité que l'on cherche à séduire et dont on attend des récompenses et des sanctions. La question de l'imaginaire de l'inspection est peu étudiée par les chercheurs, elle est pourtant d'un grand intérêt pour mieux comprendre cette situation particulière et la dégager d'un surinvestissement affectif qui obère sont efficacité.

\subsubsection{L'évaluation par le chef d'établissement}

Le professeur est aussi évalué, chaque année, par le chef d'établissement qui doit poser une note dite « «administrative » prenant trois critères en compte: ponctualité/assiduité, activité/efficacité, autorité/rayonnement. Cette note est complétée par un avis rédigé de 
quelques lignes sur la manière de servir. Ces notes sont très encadrées en fonction de l'échelon du professeur et, dans la pratique, laissent peu de marge au chef d'établissement. Néanmoins la notation et l'appréciation annuelle sont l'occasion de porter un jugement écrit sur la manière de servir à laquelle les professeurs sont sensibles. L'évaluation par le chef d'établissement rappelle ce que l'institution attend de son agent, qu'il soit présent et ponctuel, qu'il s'investissement pleinement dans son travail, avec autorité et rayonnement. Ce dernier critère a donné lieu à de nombreuses polémiques mais il est resté en vigueur et exprime la capacité à conduire une classe avec talent. Ce moment d'évaluation peut être l'occasion d'alerter le professeur sur des insuffisances, ou à l'opposé de reconnaitre un service exceptionnel. Cette note du chef d'établissement (40/100) contribue à l'avancement du professeur, mais a moins d'impact que la note portée par l'inspecteur (60/100) à l'issue d'une visite d'inspection.

\subsection{Former}

L'évaluation ne saurait être dissociée de la formation des professeurs. Il importe de distinguer la formation initiale dans les École du professorat et de l'éducation et la formation continue.

\subsubsection{Formation initiale dans les Espé}

Les Espé ont succédé en 2013 aux instituts universitaires de formation des maitres (IUFM). Actuellement le professeur admis à un concours est en formation pendant un an avant d'être titularisé. Il est astreint à un demi-service de cours devant élèves, soit 9 heures hebdomadaires pour un professeur certifié, et est suivi par deux tuteurs, l'un de l'Espé et un autre collègue chevronné de la même discipline exerçant généralement dans le même établissement que lui. Cette formation est composée d'un tronc commun et de formations liées à la discipline. Il est difficile, à ce jour, de porter un jugement sur les formations de ces très jeunes Écoles au regard des deux siècles de l'institution scolaire en France. Les Espé offrent la réelle opportunité d'une vraie formation didactique et pédagogique, au service d'un métier dont la difficulté est ressentie comme croissante, compte tenu des évolutions de la société et du développement des nouvelles technologies.

\subsubsection{La formation continue des professeurs}

Elle est sous la responsabilité des inspecteurs ${ }^{20}$, qui sont maîtres du plan de formation pour leur discipline. Les formations proposées visent à accompagner les réformes, à conforter le niveau scientifique des professeurs, à intensifier leur réflexion didactique et pédagogique pour améliorer leurs pratiques. Les inspecteurs, qui connaissent finement les professeurs et leurs besoins de formation, élaborent le plan académique de formation (PAF). Ils sont aidés par les ingénieurs de formation des rectorats et les services de formation des inspections académique qui gèrent la logistique considérable de la formation des $839700^{21}$ professeurs des écoles et

\footnotetext{
${ }^{20}$ La circulaire de 2015 réaffirme clairement la place des inspecteurs dans la formation initiale (Espé) et continue des professeurs.

${ }^{21}$ Source : Direction de l'évaluation, de la prospective et de la performance (2015), Repère et références statistiques 2015, édité par le ministère de l'éducation nationale de l'enseignement supérieur et de la recherche.
} 
établissements du second degré. La formation continue des professeurs est un enjeu considérable pour l'évolution du système éducatif. Des moyens importants lui ont été attribués à l'occasion de la réforme des collèges, chaque professeur de collège devant suivre cinq journées de formation pour la mise en place de cette réforme. La formation continue des professeurs est en pleine mutation, le rôle des Espé dans ces processus de formation doit être précisé, la mise en place des nouvelles Régions académiques, suite à la réduction du nombre de Régions françaises conduira à repenser l'offre de formation pour une plus grande rationalisation des formations offertes.

Les pratiques de GRH du système éducatif français restent centralisées et témoignent d'une certaine viscosité organisationnelle. Les évolutions dans la GRH sont lentes et des voies se sont élevées (Pochard 2008, IGEN 2013) pour réformer les pratiques de GRH qui restent l'objet d'un équilibre délicat entre le poids syndical et des tentatives réformistes souvent dénoncées comme «managériales ». Il en résulte une grande stabilité dans les pratiques qui semblent faire consensus, mais ne manquent pas d'être régulièrement interrogées par les évaluations internationales lorsqu'elles rappellent la perfectibilité du système éducatif français.

\section{Perspectives de progrès pour l'évaluation des professeurs}

Cette contribution ne saurait faire l'économie de pistes d'évolution pour l'évaluation des professeurs, de multiples préconisations ont déjà été formulées (Pochard, 2008 ; IGEN, 2013), nous retiendrons plus spécifiquement trois points essentiels, en soulignant pour conclure qu'aucun rapport ni qu'aucune recherche ne fera changer les choses sans une volonté politique forte, éclairée et réaliste.

\subsection{Pour une inspection individuelle bienveillance, exigeante et} d'accompagnement

L'inspection individuelle est une pratique qui a du sens, à la condition d'être exercée avec bienveillance et de permettre un véritable accompagnement du professeur, comme cela est posé dans la circulaire de 2015. L'imaginaire de l'institution scolaire française est habité d'inspecteurs redoutables, autoritaires et castrateurs, cherchant la faute et sanctionnant tout écart par rapport à la norme. Cette conception de l'évaluation est blessante et inefficace. L'acte d'inspection doit permettre d'évaluer les compétences disciplinaires, didactiques et pédagogiques des professeurs, avec l'exigence que l'Etat est en droit d'attendre de ceux à qui il confie la mission de la plus haute importance d'éduquer, d'instruire et de former les générations future et de répondre au défi du remplacement des hommes par les hommes (Arendt, 1961). Les audits d'établissement, de réseaux, les évaluations disciplinaires sont très utiles pour le pilotage du système éducatif, au service des recteurs et des inspecteurs d'académie. En revanche, ils ne peuvent se substituer à l'évaluation individuelle du professeur impactant effectivement le déroulement de sa carrière et permettant de prescrire des formations qui consolident ses compétences. La mise en place d'un véritable accompagnement des professeurs par les inspecteurs suppose que leur nombre soit suffisant, 
que leurs missions soient clairement définies et recentrées, et qu'ils soient formés à aux nouvelles pratiques professionnelles que l'on attend d'eux.

\subsection{Le développement professionnel des professeurs et de l'encadrement}

Le passage de la notion de formation à celle de développement professionnel permettra de donner plus de sens à l'acte d'inspection en permettant une prescription précise de formation au professeur afin qu'il progresse dans sa réflexion et sa pratique professionnelle, en échange et en lien avec ses collègues (Muller et Normand, 2013). L'encouragement de l'innovation ${ }^{22}$, la mise en place de groupes d'analyse de pratiques, la mutualisation des pratiques innovantes avec l'aide des moyens numériques, conduiront à une amélioration des pratiques enseignantes, à la réduction des tensions liées au métier (Blanchard-Laville, 2013) à l'accroissement des acquis des élèves et à la motivation des enseignants. De même le développement professionnel des chefs d'établissement et des inspecteurs tant dans la formation initiale de l'ESENESR que dans la formation continue en académie devra permettre la mise en place de groupes de mutualisation, d'analyse des pratiques de l'encadrement, de construction de compétences professionnelles tout au long de la carrière, qui confortent le sens des métiers d'encadrement et contribuent à la croissance professionnelle et personnelle de leurs acteurs (Cniré, 2014).

\subsection{Valoriser les meilleurs professeurs et accompagner les professeurs en} difficulté.

Cette question est au cour de la GRH de toute organisation et a donné lieu à de nombreux écrits récents sur une thématique vive de la GRH : le management des talents (Plane, 2015). L'éducation nationale française ne manque pas de «talents », mais elle peine à bien les gérer. D'ailleurs le terme de «talents » n'est jamais employé dans la gestion des personnels, qui n'est pas encore, tout à fait, une gestion des ressources humaines. Héritée d'une longue histoire syndicale toutes les décisions importantes en matière de gestion des personnels font l'objet de commissions paritaires - administration/syndicats - qui valident toutes les décisions en matière d'affectation, d'avancement et de mutation. Cette gestion repose sur de subtils compromis entre la valorisation des professeurs les mieux notés et les partisans d'une égalité de promotion de tous les professeurs progressant tous au même rythme, et dont la progression de carrière ne serait liée qu'à l'ancienneté. A sein des rectorats les professeurs en difficulté sont suivis par une équipe, qui cherche des solutions de remplacement et d'aménagement de service. Des tutorats sont prévus pour aider les professeurs qui en ont besoin dans la conduite de leurs classes. Force est de constater que si le suivi des professeurs en difficulté s'est amélioré ces dernières années, il reste difficile de valoriser les meilleurs professeurs, car les processus d'avancement par la notation restent très encadrés par des notes contraintes, et l'accès à la hors-classe est devenue une poursuite de carrière, quasiment automatique, une fois le dernier échelon atteint. Les pratiques d'avancement des personnels résultent d'un accord tacite entre l'administration et les syndicats afin de résoudre la tension toujours présente dans

\footnotetext{
${ }^{22}$ Cf. Rapport (2014) du Conseil National de l'Innovation pour la Réussite Educative, accessible en ligne sur le site Eduscol.
} 
toute organisation entre valorisation des meilleurs et protection des plus fragiles. Toute évolution en la matière ne peut résulter que d'un changement de mentalités qui se traduira par une évolution des rapports de pouvoir au sein de l'institution.

\subsection{L'évaluation est une question politique et non technique}

Le chercheur ne peut que s'étonner devant les analyse approfondies, les rapports officiels et les recommandations d'instances de haut niveau pour étayer une réforme de l'évaluation des professeurs qui n'a jamais eu lieu, car, en réalité, rien n'a vraiment changé dans l'évaluation des professeurs depuis quarante-quatre ans. Ainsi la lecture du rapport de Chassard et Jeanbrau (2002) remis au Haut Conseil de l'évaluation de l'école (HCÉÉ) montre un travail très abouti dans la mise en œuvre, assorti d'un calendrier de réformes... il ne sera jamais appliqué. Le décret du 7 mai $2012^{23}$ était d'une ampleur considérable, mais ce décret pris par un ministre sur le départ a été immédiatement abrogé par son successeur. Plus près de nous le rapport de l'IGEN d'avril 2013 proposait des pistes intéressantes sur l'évaluation des enseignants... qui n'ont pas été suivies de mesures effectives. L'évaluation des professeurs est donc une question très sensible et politiquement risquée. Cette pérennité ne saurait seulement s'expliquer par l'immobilisme liée aux institutions de très grande taille. Malgré son archaïsme régulièrement dénoncé, l'évaluation actuelle des professeurs est plus subtile qu'elle n'y paraît. Elle repose sur une double évaluation administrative et pédagogique, qui permet un dialogue avec les autorités hiérarchiques, chef d'établissement et inspecteur. Cette double tutelle, propre aux organisations matricielles, présente l'avantage majeur pour le professeur de pouvoir faire appel à l'un ou l'autre de ses responsables hiérarchiques en cas de difficulté. Cette possibilité de double recours est rassurante et contribue à pacifier les relations entre agents au sein d'une structure bureaucratique wébérienne (Weber, 1921; Enriquez, 1983) comme l'est l'École française. La double évaluation, malgré ses défauts, procède de la même logique et les professeurs et leurs syndicats se sont montrés très inquiets devant le projet de 2012 de faire évaluer les professeurs par le seul chef d'établissement. Toute tentative de modifier, même faiblement le processus actuel fait l'objet d'une grande vigilance de la part des syndicats qui expriment la forte sensibilité des professeurs relativement à une pratique qui impacte leur statut, leur carrière, leur rémunération et les différentes opportunités qui peuvent leur être offertes. Toute décision politique à venir en matière d'évaluation devra prendre en compte cette sensibilité à un processus qui est au cœur du fonctionnement de l'institution. La décision de modifier l'évaluation des professeurs est éminemment politique et il faudra un réel talent politique à celui, ou à celle, qui décidera de la changer.

$$
\text { - o - }
$$

\footnotetext{
${ }^{23}$ Décret $n^{\circ}$ 2012-702 du 7 mai 2012 portant dispositions statutaires relatives à l'appréciation et à la reconnaissance de la valeur professionnelle de certains personnels enseignants, d'éducation et d'orientation relevant du ministre chargé de l'éducation nationale, abrogé par le décret n 2012-999 du 27 août 2012.
} 


\section{Bibliographie}

Albanel X. (2009), Le travail d'évaluation - l'inspection dans l'enseignement secondaire, Toulouse, éd. Octarès, tiré de la thèse de l'auteur soutenue en 2007.

Arendt H. (1961), Condition de l'homme moderne, trad. G. Fradier, Paris, Calmann-Lévy, réédition, 1983, préface de Paul Ricœur ; éd. poche, Paris, Presses-Pocket, 1988, 1992.

Bachelard G. (1938), La formation de l'esprit scientifique, Paris, Vrin.

Berne E. (1971), Analyse transactionnelle et psychothérapie, traduction française par Sylvie Laroche, 1971,1977, 2001, Paris, éd. Payot.

Blanchard-Laville C. (2013), Au risque d'enseigner, Paris, Presses Universitaires de France.

CNIRÉ (2014), Rapport remis à la ministre de l'éducation nationale de l'enseignement supérieure et de la recherche, disponible en ligne sur le site Eduscol.

Crozier M. et Friedberg E. (1977), L'acteur et le système, Paris, Seuil.

Direction de l'évaluation, de la prospective et de la performance (2015), Repère et références statistiques 2015, édité par le Ministère de l'Éducation nationale de l'Enseignement supérieur et de la Recherche

Doriath B., Montaigu R., Poncelet Y., Richon H.-G. (2013), L'évaluation des enseignants, Rapport de l'inspection générale de l'éducation nationale (IGEN), publié par le ministère de l'éducation nationale, accessible en ligne.

Enriquez E. (1983), De la horde à l'Etat, essai de psychanalyse du lien social, Paris, Gallimard, collection Connaissance de l'inconscient.

Fouquet A. (2013), L'évaluation des politiques publiques, in Économie et Management, entretien avec Vinard P. et Kéradec H., n 146, janvier 2013, Futuroscope, éd. Scéren-CNDP.

Kéradec H. (2012), Epistémologie et didactique de la gestion - Le cas du concept de décision, Paris, Conservatoire national des arts métiers - thèse consultable en ligne.

Le Moigne J-L. (1974), Les systèmes de décision dans les organisations, Paris, PUF.

Muller F. et Normand R. (2013), École, la grande transformation? Les clés de la réussite, Paris, ESF éditeurs.

Plane J.-M. (2015), Théories du leadership, Paris, éd. Dunod, Collection Management Sup.

Pochard M. (2008), Livre vert sur l'évolution du métier d'enseignant, rapport au ministre de l'éducation nationale, janvier 2008, consultable en ligne.

Weber M. (1921), Économie et société, tome 1 : Les catégories de la sociologie, 1ère édition, en français, traduction du tome 1 par Julien Freund, Plon, 1971 ; dernière édition française, Paris, Pocket 2003.

Hervé Kéradec, keradec@gmail.com, février 2016. 